\title{
Design of All-Optical Nonlinear Functionalities Based on Resonators
}

\author{
Gino Priem, Student Member, IEEE, Inge Notebaert, Bjorn Maes, Student Member, IEEE, \\ Peter Bienstman, Member, IEEE, Geert Morthier, Senior Member, IEEE, and Roel Baets, Senior Member, IEEE
}

\begin{abstract}
The design of resonator-based Kerr-nonlinear functionalities for all-optical signal processing is investigated based on an analytical one-dimensional model. The influence of different device parameters is analyzed and aspects of design optimization are discussed. It is shown that important tradeoffs are to be made in terms of power budget, device length, and signal bandwidth, making these functionalities for the moment only feasible for dense wavelength-division multiplexing systems. Several issues concerning nonlinear integration in standard technologies are highlighted.
\end{abstract}

Index Terms-Bistability, Kerr nonlinearity, limiting, phase shifting, resonators, switching.

\section{INTRODUCTION}

$\mathbf{N}$ ONLINEAR optics shows great possibilities for all-optical signal processing, because of the ultrafast response times involved. The practical use of phenomena like the Kerr effect is, however, severely limited by the need for high input powers or long device lengths, since these effects are typically very small in common semiconductor systems $\left(n_{2} \approx 10^{-15}\right.$ $\left.10^{-13} \mathrm{~cm}^{2} / \mathrm{W}\right)$.

A possible way to remove these constraints is to use structures which show resonant behavior. The electric field is enhanced and the pulse is slowed down, so the nonlinear response is larger. A few examples of such structures are coupled cavity waveguides, ring resonators, etc. It has been shown that these components exhibit large improvements for the purpose of Kerrnonlinear phase shifting [1]-[5]. In addition, they also show features which cannot be implemented with plain waveguides, like all-optical limiting [6], all-optical switching, and bistability [7]-[12].

A major issue, however, is which input powers and device lengths are needed. Increasing the resonance effect will obviously result in lower power requirements, however, at the expense of the achievable signal bandwidth because increasing the resonating effect will lead to a higher finesse. This means that a tradeoff between input power, device length, and signal bandwidth will be necessary.

\footnotetext{
Manuscript received December 16, 2003; revised May 14, 2004. This work was supported in part by the Belgian DWTC IAP-PHOTON project. The work of G. Priem and B. Maes is supported in part by doctoral fellowships from the Flemish Fund for Scientific Research (FWO-Vlaanderen). The work of P. Bienstman is supported in part by a postdoctoral fellowship from the Flemish Fund for Scientific Research (FWO-Vlaanderen).

The authors are with the Department of Information Technology, Ghent University, Ghent B9000, Belgium (e-mail: gino.priem@ugent.be).

Digital Object Identifier 10.1109/JSTQE.2004.835314
}

In [1], a detailed analysis of one-dimensional (1-D) coupled resonators was performed by us to investigate this problem in the case of Kerr-nonlinear phase shifting. A 1-D model allowed us to make an analytical description of the nonlinear effects inside the structure, based on which design optimization was possible. Although this model essentially considers a nonlinear Fabry-Pérot cavity [12], it is here used in the context of waveguide implemented devices. Special attention will be paid to the limited index contrast which is typically possible in the cavity mirrors of such structures and the effect of this on the nonlinear behavior. General design aspects were obtained which are representative for all three-dimensional (3-D) structures mentioned above.

In this paper, this analysis will be extended to functionalities which are typical for photonic bandgap structures, such as all-optical limiting, switching, and bistability. All-optical limiting is the phenomenon in which the output intensity monotonically increases for increasing input power up to a limiting value and then remains more or less constant. All-optical bistability is the existence of two stable nonlinear solutions for a single input power. All-optical switching in the strict sense used in this paper is switching between zero and unity transmission by means of nonlinear interaction (and is to be distinguished from all-optical switching by means of nonlinear phase shifting in a interferometric structure). Two situations are possible: total transmission in the linear case and in the nonlinear case. These applications will be discussed in the case that the data itself behaves either linearly or nonlinearly (in contrast to pump-probe operation).

The organization of this paper is as follows. In Sections II and III, the resonating structure that will be used and the general theory from [1] will be reviewed. After that, the different nonlinear functionalities will be discussed one by one: all-optical phase shifting in Section IV, all-optical limiting in Section V, and all-optical switching and bistability in Section VI. Finally, conclusions will be drawn in Section VII.

\section{1-D STRUCTURE}

The resonator structure that was used in [1] and that will also be used in this paper has the following period:

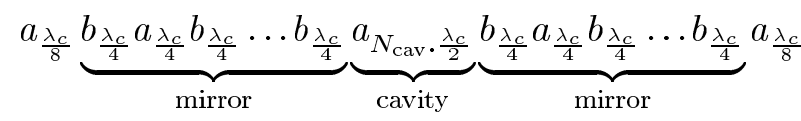

where $a$ and $b$ are two different materials, $\lambda_{c}$ is the resonance wavelength, and $N_{\text {cav }}$ is an integer indicating the cavity length (in units of $\lambda_{c} / 2$ ). The parameter $N_{\mathrm{dbr}}$ will be used to indicate 


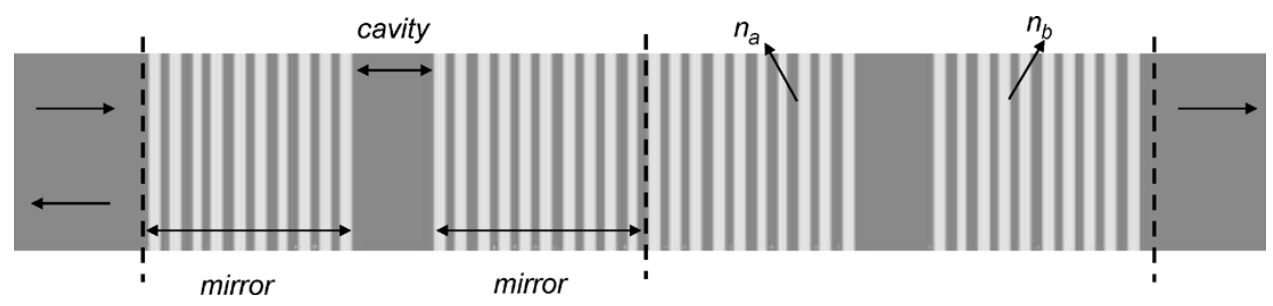

Fig. 1. Two-period resonator with in- and out-coupling layers. Refractive indices are indicated.

the total number of $b$ layers per period. The length of a single resonator period is then

$$
L_{\mathrm{res}}=\frac{\lambda_{c}}{4 n_{a}}\left(N_{\mathrm{dbr}}-1+2 N_{\mathrm{cav}}\right)+\frac{\lambda_{c}}{4 n_{b}}\left(N_{\mathrm{dbr}}\right) .
$$

Quarter-wave mirrors were chosen here for analytical purposes and because they have the largest reflectivity per unit of length. The rightmost layer of one period combines with the leftmost of the next period to create an uninterrupted distributed Bragg reflector (DBR) mirror. The homogeneous inand out-coupling layers of the resonator structure are assumed to have refractive index $n_{a}$.

An example for two periods is given in Fig. 1.

\section{THEORY}

In this section, both linear and nonlinear properties are derived for the case of resonator structures with one period and an infinite number of periods (see [1] for further details). For the general case of $N$ periods, the linear properties (complete transmission and phase relation) can be derived analytically [13], [14]. The complete nonlinear interaction for $N$ periods is much tougher to describe; however, the limiting cases with one and an infinite number of periods provide on average a good boundary, especially around the resonance wavelength for which different periods are only slightly coupled.

If the frequency dependence of the amplitude of the mirror transmission and reflection coefficients $t_{\mathrm{dbr}}$ and $r_{\mathrm{dbr}}$ is neglected and the frequency dependence of the phase is expanded linearly, the linear intensity transmission $\left|t_{\text {tot }}(\nu)\right|^{2}$ and phase relation $\phi(\nu)$ for one resonator period can be written as

$$
\begin{aligned}
\left|t_{\mathrm{tot}, \mathrm{L}}(\nu)\right|^{2} & \approx \frac{1}{1+\left|\frac{d \phi_{L}}{d \nu}\right|_{\nu_{c}}^{2}\left(\nu-\nu_{c}\right)^{2}} \\
\phi_{L}(\nu) & \approx \pm \frac{\pi}{2}-\arctan \left(\left|\frac{d \phi_{L}}{d \nu}\right|_{\nu_{c}}\left(\nu-\nu_{c}\right)\right)
\end{aligned}
$$

where the sign of $\phi_{L}(\nu)$ depends on $N_{\mathrm{dbr}}$ and

$$
\begin{aligned}
& \left|\frac{d \phi_{L}}{d \nu}\right|_{\nu_{c}}=\frac{\pi}{2 \nu_{c}\left|t_{\mathrm{dbr}}\right|_{\nu_{c}}^{2}} \\
& \quad \times\left(\left(1+\left|r_{\mathrm{dbr}}\right|_{\nu_{c}}^{2}\right)\left(2 N_{\mathrm{cav}}-1\right)+2\left|r_{\mathrm{dbr}}\right|_{\nu_{c}} \frac{n_{a}+n_{b}}{n_{a}-n_{b}}\right) .
\end{aligned}
$$

$\left|t_{\mathrm{dbr}}\right|$ and $\left|r_{\mathrm{dbr}}\right|$ represent the amplitudes of the mirror coefficients (which were assumed to be constant over the frequency)

$$
\begin{aligned}
\left|t_{\mathrm{dbr}}\right|_{\nu_{c}} & =\frac{2 n_{a}^{\frac{N_{\mathrm{dbr}}}{2}} n_{b}^{\frac{N_{\mathrm{dbr}}}{2}}}{n_{a}^{N_{\mathrm{dbr}}}+n_{b}^{N_{\mathrm{dbr}}}} \\
\left|r_{\mathrm{dbr}}\right|_{\nu_{c}} & =\frac{n_{a}^{N_{\mathrm{dbr}}}-n_{b}^{N_{\mathrm{dbr}}}}{n_{a}^{N_{\mathrm{dbr}}}+n_{b}^{N_{\mathrm{dbr}}}} .
\end{aligned}
$$

In the case of an infinite number of periods, the linear dispersion relation is approximately given by

$$
\nu-\nu_{c}=-\frac{\Delta \nu}{2} \sin \left(k L_{\mathrm{res}} \pm \frac{\pi}{2}\right)
$$

with $\Delta \nu$ the resonator bandwidth and $k L_{\text {res }}$ the phase change between the input and output over a single period, denoted as $\phi_{L}$ in the case of a resonator with a single period. From this, one can obtain

$$
\left|\frac{d \phi_{L}}{d \nu}\right|_{\nu_{c}}=\frac{2}{\Delta \nu}
$$

so finally

$$
\phi_{L}(\nu)= \pm \frac{\pi}{2}-\arcsin \left(\left|\frac{d \phi_{L}}{d \nu}\right|_{\nu_{c}}\left(\nu-\nu_{c}\right)\right) .
$$

Note that (3) and (9) can be written around $\nu=\nu_{c}$ as

$$
\phi_{L}(\nu)= \pm \frac{\pi}{2}-\left(\left|\frac{d \phi_{L}}{d \nu}\right|_{\nu_{c}}\left(\nu-\nu_{c}\right)\right) .
$$

In the presence of Kerr-nonlinear effects, the incoming light will build up inside the cavity and partially in the mirrors and, thus, alter the refractive index of the complete structure. This means that both the resonance peak can shift and resonance bandwidth can change. This index change will also modify the output phase relation $\phi(\nu)$. In the most general case, one has $n_{a, 2} \neq n_{b, 2}$. The resonance shift is then roughly determined by the overall value of $n_{2}$ (a shift to higher frequencies occurs for $n_{2}<0$ and vice versa), while the change of bandwidth is due to the modulation of $n_{2}$.

In [1], it was assumed that $n_{a, 2} \approx n_{b, 2}$, which is a good approximation for most resonator types (especially for coupled cavity waveguides). This means that the resonance bandwidth can be considered to be constant (only in cases in which $n_{a, 2}$ and $n_{b, 2}$ have opposite signs, the change of bandwidth really becomes important). A method to investigate the situation $n_{a, 2} \neq$ $n_{b, 2}$ was also described. In this paper, the same assumptions will be used. 
The shift of the linear resonance frequency $\nu_{c, L}$ is then

$$
\Delta \nu_{c} \approx-\frac{3}{4}\left(\frac{n_{a, 0}}{n_{b, 0}}\right)^{N_{\mathrm{dbr}}}\left|E_{\mathrm{in}}\right|^{2} \frac{n_{2}}{n_{a, 0}} \nu_{c} \frac{N_{\mathrm{cav}}+\frac{n_{a, 0}^{4}+n_{b, 0}^{4}}{n_{a, 0}^{4}-n_{b, 0}^{4}}}{N_{\mathrm{cav}}+\frac{n_{b, 0}}{n_{a, 0}-n_{b, 0}}}
$$

in the case $n_{a, 0}>n_{b, 0}$, which is always the case for resonators. Note that this shift holds for any number of periods, because when $\left|t_{\text {tot }}\right|^{2}=1$, each period is independent.

The frequency shift for the linear frequency $\nu \neq \nu_{c, L}$ will not be equal to $\Delta \nu_{c}$. In general, the field profile of one resonator period for a certain frequency $\nu$ will scale more or less with a factor $\left|t_{\text {tot,L }}(\nu)\right|$ compared to $\nu_{c, L}$. And since the Kerr effect scales with $|E|^{2}$, the shift will now be

$$
\Delta \nu_{\mathrm{NL}} \approx\left|t_{\mathrm{tot}, \mathrm{L}}(\nu)\right|^{2} \Delta \nu_{c}
$$

which means that the Kerr-nonlinear intensity transmission for one period is approximately given by

$$
\left|t_{\mathrm{tot}, \mathrm{NL}}\left(\nu^{\prime}\right)\right|^{2}=\left|t_{\mathrm{tot}, \mathrm{L}}(\nu)\right|^{2}
$$

with $\nu^{\prime}=\nu+\left|t_{\text {tot }, \mathrm{L}}(\nu)\right|^{2} \Delta \nu_{c}$. In the same way, and since the bandwidth was assumed to be constant, the phase relation becomes

$$
\phi_{\mathrm{NL}}\left(\nu^{\prime}\right)=\phi_{L}(\nu)
$$

with

$$
\left|\frac{d \phi_{L}}{d \nu}\right|_{\nu_{c, L}} \approx\left|\frac{d \phi_{\mathrm{NL}}}{d \nu}\right|_{\nu_{\mathrm{c}, \mathrm{NL}}} \equiv\left|\frac{d \phi}{d \nu}\right|_{\nu_{c}}
$$

For all-optical phase shifting, the transmission must be close to unity $\left(\left|t_{\text {tot }}(\nu)\right|^{2} \approx 1\right)$, so the phase shift will in this case be

$$
\Delta \phi \approx 2 \arctan \left(\left|\frac{d \phi}{d \nu}\right|_{\nu_{c}} \frac{\Delta \nu_{c}}{2}\right)
$$

For an infinite number of periods, these problems do not arise and the transmission window simply shifts, so

$$
\phi_{\mathrm{NL}}(\nu)= \pm \frac{\pi}{2}-\arcsin \left(\left|\frac{d \phi}{d \nu}\right|_{\nu_{c}}\left(\nu-\nu_{\mathrm{c}, \mathrm{NL}}\right)\right) .
$$

In the bistable region, in fact, two phase relations exist: the "transmissive" one is given here; the other one is simply $\phi_{\mathrm{NL}}(\nu)=\pi$ if $n_{2}>0$ and $\phi_{\mathrm{NL}}(\nu)=0$ if $n_{2}<0$. With this, $\Delta \phi$ is equal to

$$
\Delta \phi=2 \arcsin \left(\left|\frac{d \phi}{d \nu}\right|_{\nu_{c}} \frac{\Delta \nu_{c}}{2}\right)
$$

In the general case of $N$ periods, the method used for a single period (namely, $\nu^{\prime}=\nu+\left|t_{\text {tot }, \mathrm{L}}(\nu)\right|^{2} \Delta \nu_{c}$ ) cannot be repeated because the linear field for $\nu \neq \nu_{c, L}$ is different in every cavity. One could think of using an average over every cavity; however, the equations obtained in this way are very complex and not completely stable, so they provide no advantage over numerical simulations. Nevertheless, conclusions for a single period will also influence the design for structures with more periods. Only the phase shift can more or less be approximated (in the case high transmission is assumed) from (16) and (18) to be

$$
\Delta \phi=\left|\frac{d \phi}{d \nu}\right|_{\nu_{c}} \Delta \nu_{c}
$$

which is in agreement with intuitive thinking.

\section{All-Optical Phase Shifting}

In all-optical phase shifting, a certain phase shift (typically $\pi$ ) must be achieved at the device output between the case of a high-power (Kerr-nonlinear behavior) and a low-power data signal (linear behavior) for a certain data rate $\Delta \nu_{s}$.

The resonance peaks in the linear and the nonlinear case do not coincide, so the available signal bandwidth can be substantially lower than the resonance bandwidth $\Delta \nu$. In the ideal case, $\Delta \nu_{s}$ will be equal to the bandwidth overlap between both cases, thus

$$
\Delta \nu_{s}=\Delta \nu-\Delta \nu_{c}
$$

In case of a realistic, finite structure, the resonance window will in fact not be completely transmissive. At the edges, transmission peaks will appear (an increasing number for a higher number of periods), between which the transmission can drop even more than $50 \%$, depending on the index contrast of the mirrors. Therefore, $\Delta \nu$ should be corrected with a factor $f_{\text {corr }}<1$, so the maximum signal bandwidth is

$$
\Delta \nu_{s}=f_{\text {corr }} \Delta \nu-\Delta \nu_{c}
$$

It was found that $f_{\text {corr }}=0.5$ is a good estimate.

From (8), (11), (19), and (21), it is obvious that a tradeoff between different design specifications will have to be made: to minimize the device length $L_{\text {tot }}$, the phase shift per unit of length $\Delta \phi / L_{\text {res }}$ must be maximized. This implies that the resonance shift $\Delta \nu_{c}$ should be as high and the resonance bandwidth $\Delta \nu$ as low as possible. However, these last two conditions also result in a lower signal bandwidth $\Delta \nu_{s}$. Furthermore, a restriction to the resonance shift $\Delta \nu_{c}$ will be imposed by the achievable input power.

Optimization is now done for a realistic example: an coupled cavity photonic wire in silicon-on-insulator (SOI) is approximated by taking as effective indices $n_{a}=2.6$ and $n_{b}=$ 2.36 , which corresponds to a index contrast of $\approx 10 \%$. The Kerr coefficient of silicon at $1.5 \mu \mathrm{m}$ is equal to $n_{2}=0.6 \times$ $10^{-13} \mathrm{~cm}^{2} / \mathrm{W}$ (or $2.4 \times 10^{-16} \mathrm{~cm}^{2} / \mathrm{V}^{2}$ ) [15] and the cross-section area $A_{\text {cross }} \approx\left(\lambda_{c} / 2 n_{a}\right)^{2}$. The input power $P_{\text {in }}$ corresponding to a certain input field $E_{\text {in }}$ may be estimated from the input intensity $I_{\text {in }}=(1 / 2) c n_{a} \epsilon_{0}\left|E_{\text {in }}\right|^{2}$ and the cross-section area, so

$$
P_{\mathrm{in}} \approx I_{\mathrm{in}} A_{\mathrm{cross}}=\frac{c}{8 n_{a}} \epsilon_{0} \lambda_{c}^{2}\left|E_{\mathrm{in}}\right|^{2}
$$

The results are shown in Fig. 2: the expected minimal input power is shown as a function of device length for several signal 


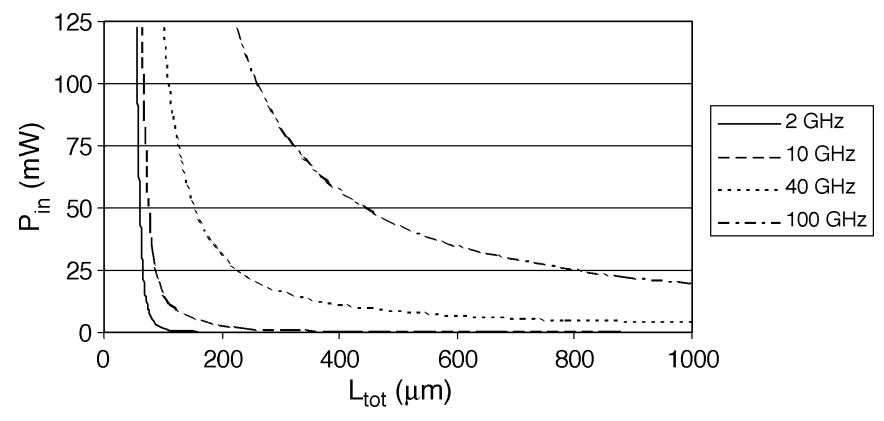

Fig. 2. Input power $P_{\text {in }}$ required to obtain a nonlinear phase shift of $\pi$ by propagation through a resonator-based distance of $L_{\text {tot }}$ for several signal bandwidths [(21), calculated from (19) and (22)].

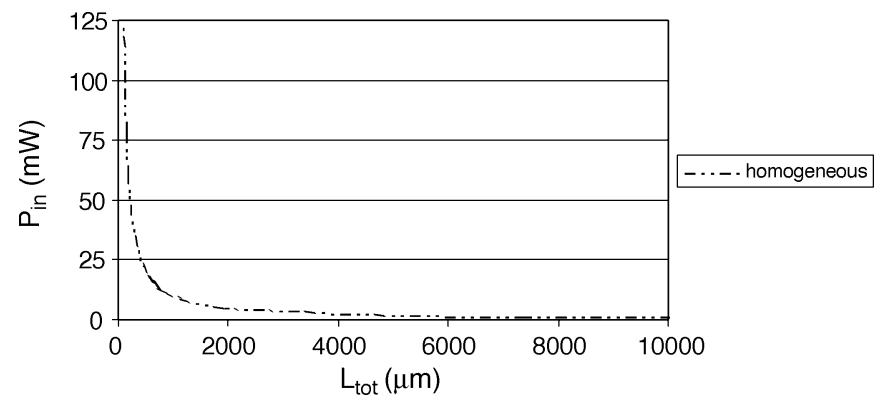

Fig. 3. Input power $P_{\text {in }}$ required to obtain a nonlinear phase shift of $\pi$ by propagation through a homogeneous distance of $L_{\text {tot }}$.

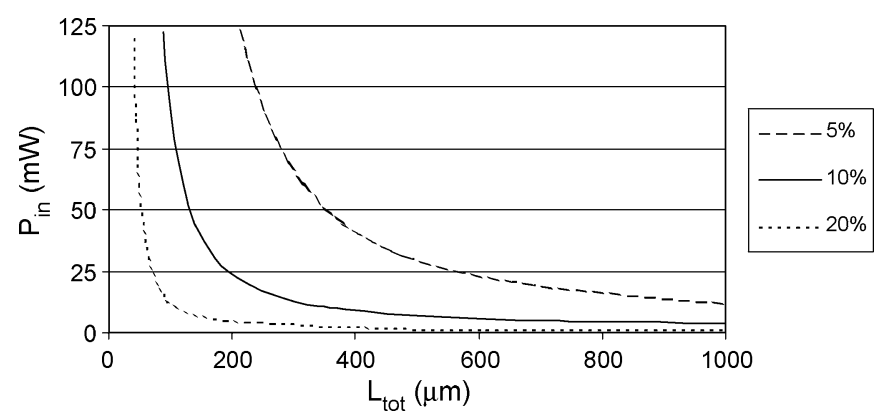

Fig. 4. Influence of index contrast on the $P_{\text {in }}-L_{\text {tot }}$ tradeoff, shown in Fig. 2, for the case of $40 \mathrm{GHz}$.

bandwidths. To give rise to smooth curves, the parameters $N_{\mathrm{dbr}}$ and $N$ were assumed to be continuous, which is a good approximation, since both values are typically high. The situation of a simple wire without resonating structures is drawn in Fig. 3 as a comparison.

Comparing Fig. 2 and Fig. 3, it can be seen that improvements for the device length in the order of 10000 are possible, depending on the signal bandwidth. The signal bandwidth is, however, an important limiting factor, especially for very high band rates. Note also that from a certain input power on, the relative improvement of $L_{\text {tot }}$ drops very steeply, so using even higher powers in that situation is not sound.

In Fig. 4, the example above is recalculated with other index contrasts for a signal bandwidth of $40 \mathrm{GHz}$. From this, it is clear that high-index contrasts allow major improvements in the device length. This is due to two factors: first of all, the length of mirrors with the same reflectivity substantially reduces for higher contrast [see (1) and (6)]. In addition, the resonance shift for a certain input power will also be larger for shorter mirrors (11), because a smaller part of the nonlinear phase change in the cavity will be used to compensate the phase shift in the mirrors, as discussed in [1]. The obtainable index contrast is, however, typically limited by scattering and radiation loss in actual structures.

Another important remark is that for all situaties depicted in Fig. 2 and Fig. 4, one has $N_{\text {cav }}=1$. From (8) and (4), it can be seen that the resonance bandwidth reduces both with higher $\left|r_{\mathrm{dbr}}\right|_{\nu_{c}}$ and larger $N_{\mathrm{cav}}$. However, only the mirror strength determines the field strength inside the cavity. On the other hand, a larger cavity improves the frequency shift, as can be seen from the cavity dependence of (11). The result that $N_{\text {cav }}=1$ is the optimum solution now implies that increasing $\left|r_{\mathrm{dbr}}\right|_{\nu_{c}}$ is more efficient in terms of device length than increasing the cavity length.

To determine the real optimum, only integer values of $N_{\mathrm{dbr}}$ and the period number $N$ must be taken into account. In most situations, one will also choose $N_{\mathrm{dbr}}$ to be even (this was implicitely done in Section II). Otherwise $b_{\lambda_{c} / 8}$ layers must be constructed at the in- and out-coupling sections, which require more demanding feature sizes. Suppose one would like to minimize the device length for a signal bandwidth of $10 \mathrm{GHz}$ and input powers up to $30 \mathrm{~mW}$ are allowed. A method to find this optimal solution was described in [1]. The best solution in this case is

$$
\left.\begin{array}{rl}
N_{\text {cav }} & =2 \\
N_{\text {dbr }} & =54 \\
N & =5 \\
P_{\mathrm{NL}} & =29.7 \mathrm{~mW}
\end{array}\right\} L_{\text {tot }}=87.2 \mu \mathrm{m} .
$$

Because of the discrete character of the solution space, $N_{\text {cav }}$ is not necessarily equal to one; it is possible that a higher $N_{\text {cav }}$ value lies closer to the continuous optimum than the situation $N_{\text {cav }}=1$. As noted above, increasing $\left|r_{\mathrm{dbr}}\right|_{\nu_{c}}$ is more efficient to reduce the device length than increasing the cavity length. However, for low cavity values, this difference in efficiency is relatively low, because increasing the cavity length still improves the frequency shift substantially. Large $N_{\text {cav }}$ values ( $>5$ ) on the other hand will rarely be the optimum, since the relative improvement of $\Delta \nu_{c}$ will drop (as can be seen from (11)).

The results obtained above are now checked numerically in Fig. 5. This was done by using a nonlinear extension [16] of the CAvity Modeling FRamework (CAMFR) [17] based on spatial index discretization.

From Fig. 5, it can be seen that the resonance shift is equal to $0.19 \mathrm{~nm}$, which is in good agreement with (11). The obtained phase shift for $\lambda=(1 / 2)\left(\lambda_{c, L}+\lambda_{c, \mathrm{NL}}\right)=1.550095 \mu \mathrm{m}$ is $\Delta\left|\phi_{\text {tot }}\right| \approx 3.32$, which is close to $\pi$. As can be seen, the phase shift is almost constant over a large area $(\approx 0.173 \mathrm{~nm})$; however, for reasons of transmission, the usable wavelength range $\left(\left|t_{\text {tot }}\right|^{2}>0.9\right)$ is only $\Delta \lambda_{s}=0.085 \mathrm{~nm}$, so the signal bandwidth is approximately limited to $10 \mathrm{GHz}$, as expected. 


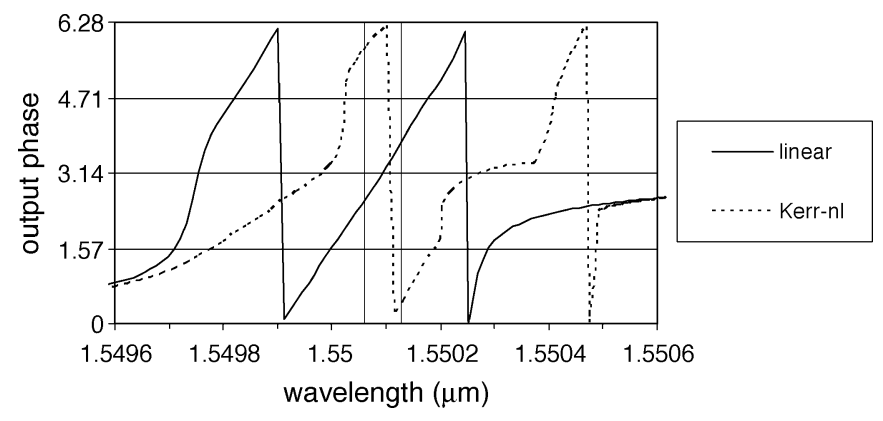

Fig. 5. Numerical calculation of the linear and nonlinear phase relation $\phi_{L}(\lambda)$ and $\phi_{\mathrm{NL}}(\lambda)$. The parameters values used can be found in the text. The signal bandwidth is indicated by vertical lines.

\section{All-OpticAl Limiting}

All-optical limiting is the phenomenon in which the incoming power $P_{\text {in }}$ is limited to a certain value which is constant for a large range of input powers $\left[P_{a}, P_{b}\right]$. This can be achieved by decreasing the total transmission with higher $P_{\text {in }}$ by means of shifting the resonance frequency.

In general, the output power $P_{\text {out }}=\left|t_{\text {tot }, \mathrm{NL}}\right|^{2} P_{\text {in }}$ will not be completely constant over the range $\left[P_{a}, P_{b}\right]$. This can be expressed by the flatness $F$ of the output power defined as

$$
F=\frac{P_{\text {out }}\left(P_{b}\right)-P_{\text {out }}\left(P_{a}\right)}{P_{\text {out }}\left(\frac{P_{a}+P_{b}}{2}\right)} .
$$

$F$ is nothing more than the output power fluctuation relative to the average output power. To obtain a steep climb to the limiting value, the signal bandwidth should be chosen around $\nu_{c, L}$ because there one has $\left|t_{\text {tot, NL }}\right|^{2} \approx 1$ for low $P_{\text {in }}$. In addition, to ensure a low pulse shape distortion, the output power should be approximately constant over the signal bandwidth $\Delta \nu_{s}$. If the frequency variation $V$ for the input power $P_{\text {in }}=\left(P_{a}+P_{b}\right) / 2$ is defined as

$$
V=\frac{P_{\text {out }}\left(\nu_{c, L}+\frac{\Delta \nu_{s}}{2}\right)-P_{\text {out }}\left(\nu_{c, L}-\frac{\Delta \nu_{s}}{2}\right)}{P_{\text {out }}\left(\nu_{c, L}\right)}
$$

a typical limit to avoid pulse distortion is given by $V<10 \%-20 \%$.

Suppose now one would like to construct a limitor for incoming powers between 60 and $180 \mathrm{~mW}$ by using the same refractive indices as in Section IV. For $V<10 \%$, the obtainable output values were calculated using (13), (23), and (24) and plotted as a function of the minimal flatness for several signal bandwidths in Fig. 6 in the case of a single resonator. $N_{\mathrm{dbr}}$ was again assumed to be continuous.

An upper and lower bound for $P_{\text {out }}$ can be seen for each obtainable $\Delta \nu_{s}$. The upper bound is equal to all $\Delta \nu_{s}$ and is only determined by $F$. The lower bound is the result of $V<10 \%$, which led to the fact that only $\Delta \nu_{s}=2 \mathrm{GHz}$ appears in Fig. 6. A relaxation of the allowed frequency variation up to $V<60 \%$ is required to allow $\Delta \nu_{s}=10 \mathrm{GHz}$ to appear into the figure. The influence of the index contrast on the obtainable output power is very small.

Optimization is now performed for the limit $P_{\text {out }}=10 \mathrm{~mW}$ and $\Delta \nu_{s}=2 \mathrm{GHz}$. To do this, the flatness is plotted as a function of $N_{\text {cav }}$ and $N_{\text {dbr }}$ in Fig. 7. Also a line is drawn for which

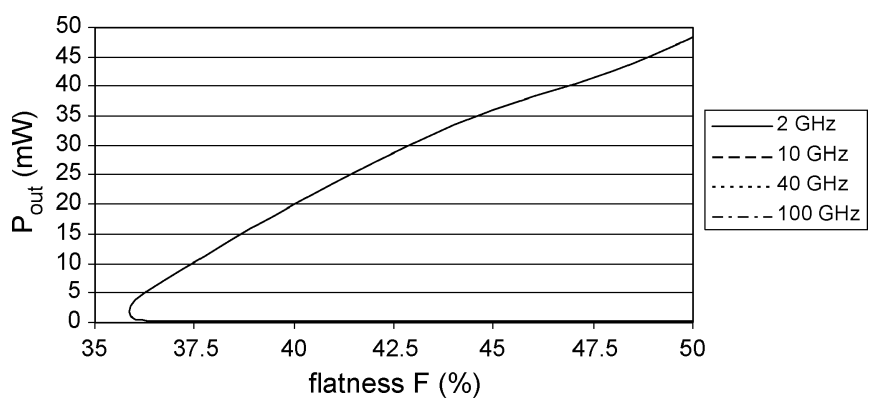

Fig. 6. Upper and lower bound for the obtainable output power limit $P_{\text {out }}\left(\left(P_{a}+P_{b}\right) / 2\right)$ as a function of the flatness of the output power curve $F$ for several signal bandwidths, obtained from (13), (23), and (24). The parameter values used are indicated in the text. The frequency variation $V$ is lower than $10 \%$.

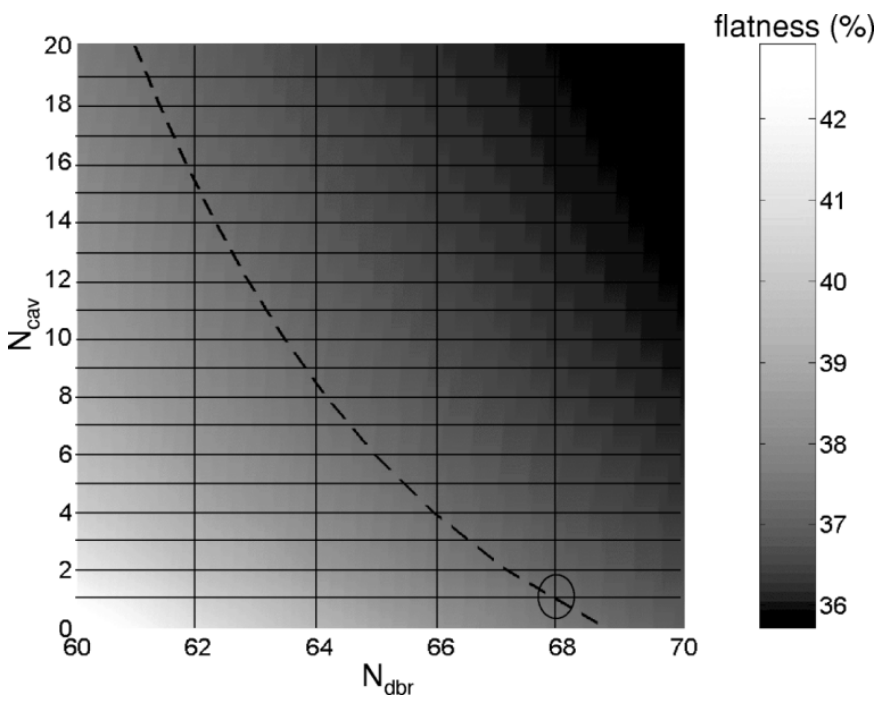

Fig. 7. Output power flatness $F$ obtainable with $N_{\text {cav }}$ and $N_{\mathrm{dbr}}$ for a signal bandwidth of $\Delta \nu_{s}=2 \mathrm{GHz}$. The dotted line corresponds to $P_{\text {out }}=10 \mathrm{~mW}$. The optimum value is indicated by a circle.

$P_{\text {out }}=\left|t_{\text {tot, } \mathrm{NL}}\right|^{2} P_{\text {in }}=10 \mathrm{~mW}$. The best parameter choice is denoted with a circle.

It can be seen that $F$ is almost constant along the line $P_{\text {out }}=$ $10 \mathrm{~mW}$ ( $F \approx 37.5 \%)$. The $N_{\text {cav }}$ value can generally be used to tune the output power exactly to the desired limit.

These results are now numerically checked in Fig. 8. Note that the scale of both axes is different. The obtained output power fluctuation is $3.72 \mathrm{~mW}$, which corresponds to $F \approx 37.2 \%$. This is in excellent agreement with the minimal flatness from Fig. 6.

Note that for the same output power, the minimal flatness and frequency variation will relax strongly for higher $P_{a}$ and $P_{b}$. This improvement can already be seen in Fig. 8: the limiting is still improving for higher input powers. But in practice, one will not want to limit the output power to more than $10 \%-20 \%$ of its original value. The importance of this remark, however, lies in the fact that increasing $P_{\text {in }}$ is equivalent to higher $n_{2}$ values. The higher the Kerr-nonlinear coefficient, the better the limiting will be.

Another remark concerns the number of periods which is to be used. In the example above, only one period was used. Nevertheless, this is the best choice, because the limiting range as 


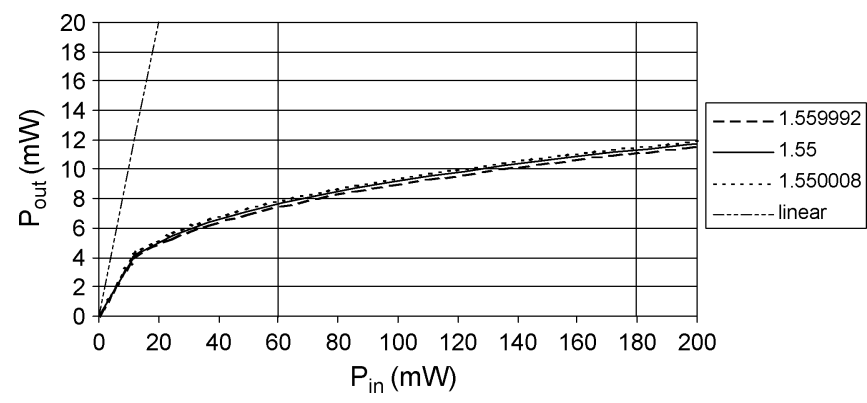

Fig. 8. Numerical calculation of output power $P_{\text {out }}$ versus input power $P_{\text {in }}$ for parameter values discussed in the text. The case of linear transmission is shown as a reference.

well as the frequency variation will deteriorate for higher $N$. Indeed, in the extreme case of an infinite number of periods, the only possible output power with an acceptable value for $V$ is $P_{\text {out }}=0$. This will also become more clear in Section VI-A.

\section{All-Optical Switching}

As mentioned in the introduction, two possible situations of all-optical switching exist: linear-high and nonlinear-low transmission on the one hand and linear-low and nonlinear-high transmission on the other. The former case is related to all-optical limiting, in the sense that both make use of a reduced transmission with increasing input intensity. However, while limiting is used for a extensive range of large input powers, all-optical switching will operate based on the fact that the incoming data signal is either low power or high power. The latter case is more related to bistability, however, trying to avoid the bistable region itself to ensure a single solution.

\section{A. Linear-High and Nonlinear-Low Switching}

To obtain a high transmission in the linear case, the signal bandwidth must be centered on the linear resonance frequency $\nu_{c, L}$. In the nonlinear situation, the high-power data signal will shift the resonance frequency and the nonlinear transmission will become low. This transmission will be lower for higher input power. The minimal transmission contrast $C$ will be given by

$$
C=\left|t_{\mathrm{tot}, \mathrm{L}}\right|^{2}-\left|t_{\mathrm{tot}, \mathrm{NL}}\right|^{2}
$$

with $\left|t_{\text {tot }, \mathrm{L}}\right|^{2}=\min \left(\left|t_{\text {tot }, \mathrm{L}}(\nu)\right|^{2}\right)$ and $\left|t_{\text {tot, }, \mathrm{NL}}\right|^{2}=$ $\max \left(\left|t_{\mathrm{tot}, \mathrm{NL}}(\nu)\right|^{2}\right)$ for $\nu \in\left[\nu_{c, L}-\left(\Delta \nu_{s} / 2\right), \nu_{c, L}+\left(\Delta \nu_{s} / 2\right)\right]$.

This contrast is optimized in function of the nonlinear input power for the same parameter values used above and for different signal bandwidths in Fig. 9, by means of (13) and (25). A structure with a single period was used. Again $N_{\mathrm{dbr}}$ was assumed to be continuous.

From the discussion above, it is already clear that a tradeoff between the achievable contrast and the input power of the nonlinear data signal is to be made. Fig. 9, however, shows that rather high powers are required to achieve reasonable transmission contrasts. And what is more, these requirements increase enormously with increasing signal bandwidth. Fig. 10 shows the high influence of the index contrast on the obtainable transmission contrast for a constant bandwidth. This is a consequence of

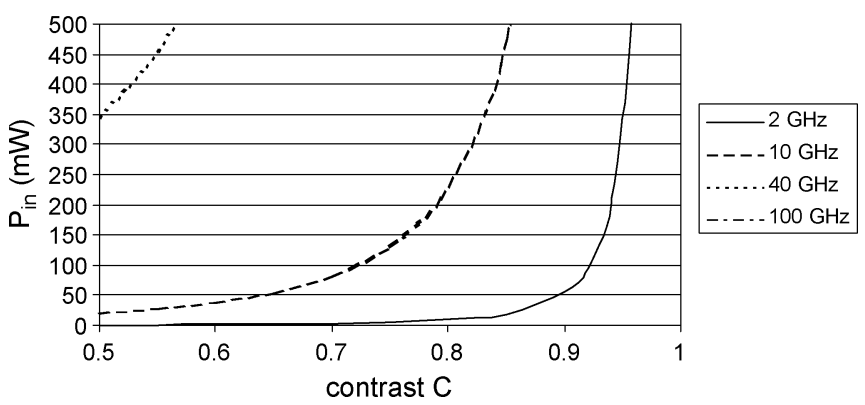

Fig. 9. Input power $P_{\text {in }}$ required to obtain a transmission contrast $C$ for various signal bandwidths, as predicted by (13) and (25).

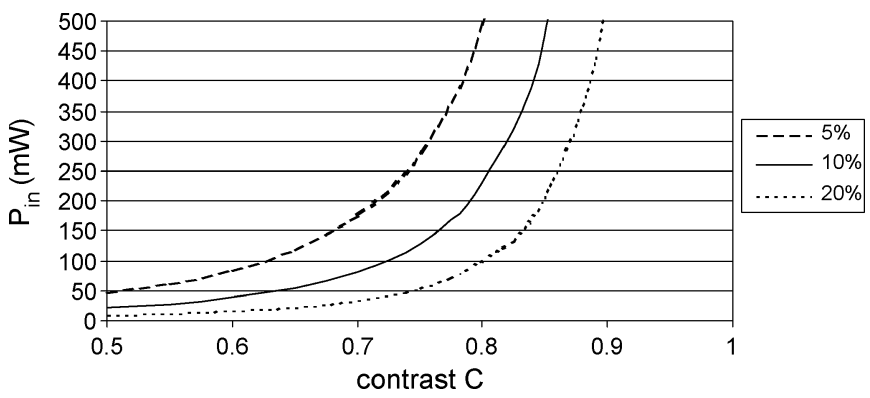

Fig. 10. Influence of index contrast on the $P_{\text {in }}-C$ tradeoff, shown in Fig. 9, for a signal bandwidth of $10 \mathrm{GHz}$.

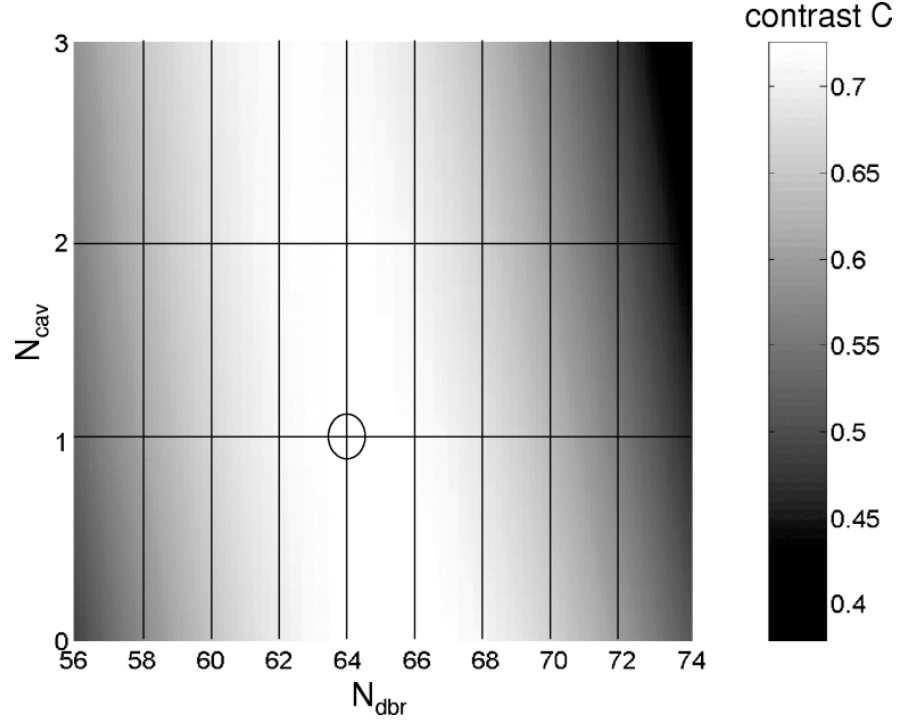

Fig. 11. Obtainable transmission contrast $C$ as a function of $N_{\mathrm{dbr}}$ and $N_{\text {cav }}$ for an input power of $P_{\text {in }}=100 \mathrm{~mW}$ and a signal bandwidth of $\Delta \nu_{s}=$ $10 \mathrm{GHz}$. The optimum value is indicated by a circle.

the index dependence of the resonance shift (11), which was already discussed in Section IV. The optimal cavity value in Figs. 9 and 10 is again $N_{\text {cav }}=1$ based on the same principles as discussed in Section IV.

Optimization is now carried out for the case in which a nonlinear input power of $P_{\text {in }}=100 \mathrm{~mW}$ is allowed and for a signal bandwidth of $10 \mathrm{GHz}$. The obtained transmission contrast for $P_{\text {in }}=100 \mathrm{~mW}$ as a function of $N_{\mathrm{dbr}}$ and $N_{\text {cav }}$ is plotted in Fig. 11.

Note that $P_{\text {in }}<100 \mathrm{~mW}$ will always result in a lower transmission contrast for the same value of $N_{\mathrm{dbr}}$ and $N_{\text {cav }}$ because 


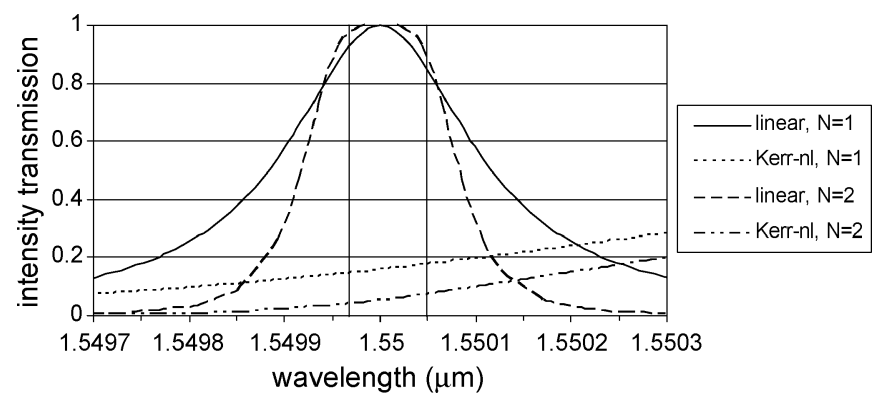

Fig. 12. Numerical calculation of the linear and nonlinear transmission $\left|t_{\text {tot }}(\lambda)\right|^{2}$ for a resonator structure consisting of one and two periods. The parameters values used can be found in the text. The signal bandwidth is indicated by vertical lines.

a lower $P_{\text {in }}$ corresponds to lower resonance shift, so only the case $P_{\text {in }}=100 \mathrm{~mW}$ must be taken into account. The highest contrast is obtained for

$$
\left.\begin{array}{rl}
N_{\text {cav }} & =1 \\
N_{\text {dbr }} & =64 \\
L_{\text {res }} & =20.3 \mu \mathrm{m}
\end{array}\right\} C=0.732
$$

which is very close to the value obtained from Fig. 9.

This is again checked numerically in Fig. 12. The linear and nonlinear transmission is plotted here and the signal bandwidth is indicated. A transmission contrast of $C=0.717$ is obtained, which is in very good agreement with the analytical result $C=$ 0.732 .

Until now, only one period was taken into account. It is, however, clear that the contrast will be increased for exactly the same parameters by simply using more resonator periods: the linear transmission $\left|t_{\text {tot, } \mathrm{L}}\right|^{2}$ will be higher and the nonlinear transmission $\left|t_{\text {tot, } \mathrm{NL}}\right|^{2}$ lower, because the resonance peak becomes more confined for higher $N$. This is also shown in Fig. 12: by using the same parameters and two periods instead of one, the transmission contrast is already improved to $C=0.864$. In the extreme case of $\infty$ periods, a contrast of unity is achieved if $\Delta \nu_{c}=\Delta \nu_{s}+\left(\Delta \nu-\Delta \nu_{s}\right) / 2=\left(\Delta \nu+\Delta \nu_{s}\right) / 2$. In the example discussed above, an input power of only $P_{\text {in }}=9.1 \mathrm{~mW}$ would be needed for this.

However, in this discussion one aspect has not been mentioned yet: the output power is not $\left|t_{\text {tot }}\right|^{2}$, but $\left|t_{\text {tot }}\right|^{2} P_{\text {in }}$. And since this type of switching is used in the same frequency range as all-optical limiting $\left(\nu \in\left[\nu_{c, L}-\left(\Delta \nu_{s} / 2\right), \nu_{c, L}+\left(\Delta \nu_{s} / 2\right)\right]\right)$, all-optical limiting is actually what really happens. This means that the linear-high transmission state will have a lower output power than the nonlinear-low transmission state (compare with Fig. 8). All-optical linear-high/nonlinear-low switching can nevertheless still be used by simply changing the data processing. In the case of one period, the output power of Fig. 12 for $P_{\text {in }}=100 \mathrm{~mW}$ is $P_{\text {out }} \approx 12 \mathrm{~mW}$. If, e.g., the nonlinear off-state is defined at the detector side as $10-15 \mathrm{~mW}$, the nonlinear on-state as $80-110 \mathrm{~mW}$, the linear off-state as $0-1 \mathrm{~mW}$ and the linear on-state as 4-7 $\mathrm{mW}$, linear-high/nonlinear-low switching is perfectly possible.

Note that this is also the reason why the number of periods must not be too high. Based on Section V and Fig. 12, one can see that for high $N$, the nonlinear off-state will come close to

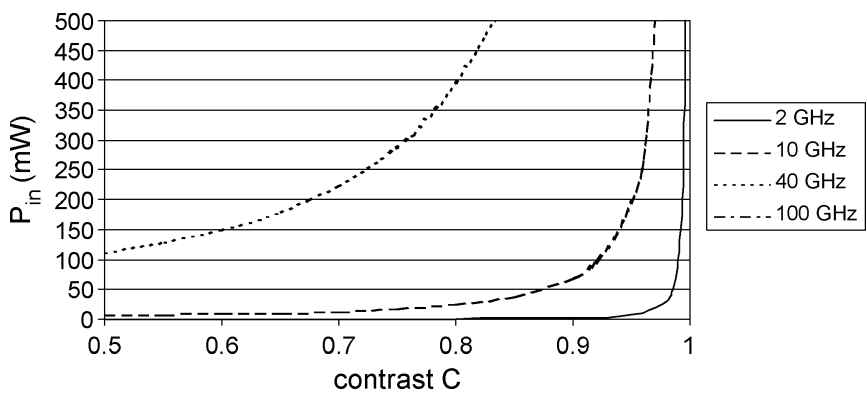

Fig. 13. Input power $P_{\text {in }}$ required to obtain a transmission contrast $C$ for various signal bandwidths, as calculated from (13) and (26).

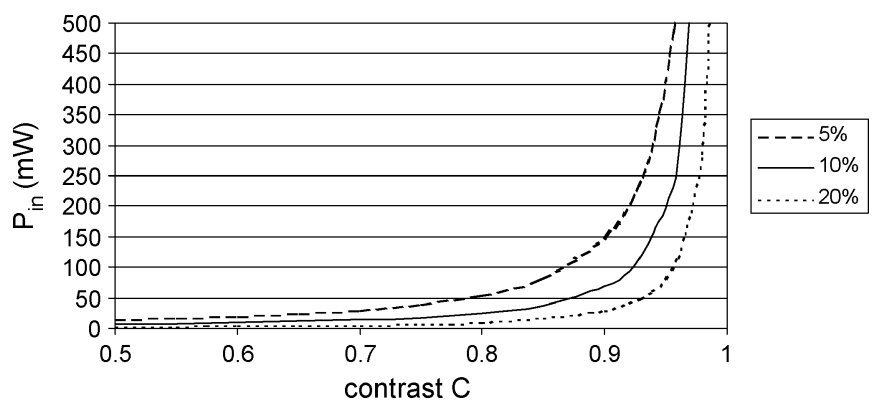

Fig. 14. Influence of index contrast on the $P_{\text {in }}-C$ tradeoff, shown in Fig. 13, for a signal bandwidth of $10 \mathrm{GHz}$.

the linear on-state and the difference will become unclear. This also means that in fact very high contrast (thus, low $\left|t_{\text {tot,NL }}\right|^{2}$ ) is not wanted. $\left|t_{\text {tot, NL }}\right|^{2}$ must only be low enough to be able to distinguish the nonlinear on- and off-state.

\section{B. Low-Linear and High-Nonlinear Switching and Bistability}

This type of switching is in many aspects the opposite of the previous type. Since the nonlinear transmission must in this case be as high as possible, the signal bandwidth will be centered on the nonlinear resonance frequency $\nu_{\mathrm{c}, \mathrm{NL}}$. The minimal transmission contrast $C$ is now defined as

$$
C=\left|t_{\mathrm{tot}, \mathrm{NL}}\right|^{2}-\left|t_{\mathrm{tot}, \mathrm{L}}\right|^{2}
$$

with $\left|t_{\text {tot, }, \mathrm{NL}}\right|^{2}=\min \left(\left|t_{\mathrm{tot}, \mathrm{NL}}(\nu)\right|^{2}\right)$ and $\left|t_{\text {tot }, \mathrm{L}}\right|^{2}=$ $\max \left(\left|t_{\text {tot }, \mathrm{L}}(\nu)\right|^{2}\right)$ for $\nu \in\left[\nu_{\mathrm{c}, \mathrm{NL}}-\left(\Delta \nu_{s} / 2\right), \nu_{\mathrm{c}, \mathrm{NL}}+\left(\Delta \nu_{s} / 2\right)\right]$.

Just like in Section VI-A, this contrast will be larger for higher input powers and the optimization of the tradeoff between contrast and input power for the same parameters as for the other functionalities is shown in Fig. 13 for the case of a single period. $N_{\text {dbr }}$ was assumed to be continuous. Fig. 14 shows the influence of the refractive index contrast.

Exactly the same conclusion can be drawn as in the case of "high-linear and low-nonlinear" switching. One can, however, see by comparing Fig. 9 with Fig. 13 that here much higher contrast can be achieved with the same power level.

The optimization is now done for the same situation as in Section VI-A, i.e., $P_{\text {in }}=100 \mathrm{~mW}$ and $\Delta \nu_{s}=10 \mathrm{GHz}$. The obtained transmission contrast as function of $N_{\mathrm{dbr}}$ and $N_{\text {cav }}$ is plotted in Fig. 15.

Again $P_{\text {in }}<100 \mathrm{~mW}$ will result in a lower transmission contrast for the same value of $N_{\mathrm{dbr}}$ and $N_{\text {cav }}$ because a lower $P_{\text {in }}$ corresponds to lower resonance shift, so only the case $P_{\text {in }}=$ 


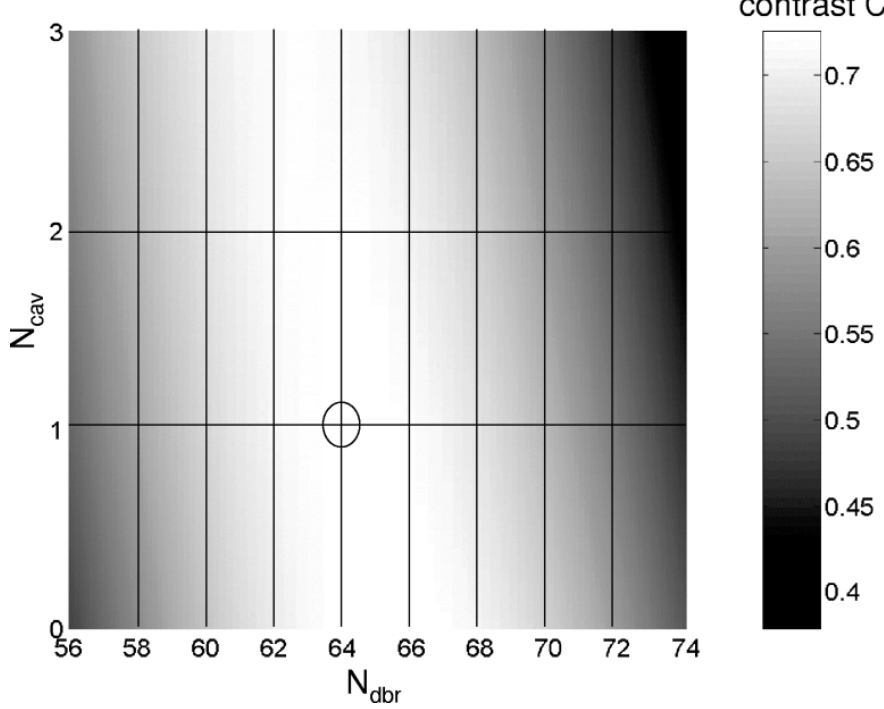

Fig. 15. Obtainable transmission contrast $C$ as a function of $N_{\mathrm{dbr}}$ and $N_{\text {cav }}$ for an input power of $P_{\text {in }}=100 \mathrm{~mW}$ and a signal bandwidth of $\Delta \nu_{s}=$ $10 \mathrm{GHz}$. The optimum value is indicated by a circle.

$100 \mathrm{~mW}$ is to be taken into account. One obtains the highest contrast for

$$
\left.\begin{array}{rl}
N_{\text {cav }} & =1 \\
N_{\text {dbr }} & =60 \\
L_{\text {res }} & =19.0 \mu \mathrm{m}
\end{array}\right\} C=0.927 .
$$

The numerical check is shown in Fig. 16. The linear and nonlinear transmission is plotted and the signal bandwidth is indicated. A transmission contrast of $C=0.930$ is achieved, in excellent agreement with the analytical prediction.

However, this figure also shows that in the region of interest, actually two stable solutions exist. This can also be seen analytically from (12) and (13): the shift of the top of the resonance peak is much larger that the shift of frequencies at the side of the peak. The major problem now is that the solution that will be excited is the one with the lowest transmission $\left(\left|t_{\text {tot, NL }}\right|^{2} \approx\right.$ $\left.\left|t_{\text {tot }, \mathrm{L}}\right|^{2}\right)$, because one is coming from $\pm 0 \mathrm{~mW}$.

One could wonder if it is not possible to achieve this type of switching without bistability. The answer is yes, but only for very low signal bandwidth $(<2 \mathrm{GHz})$ and with very low transmission contrasts $(<0.65)$. A possible way, however, to overcome this obstacle is using pulses which have a very high power density at the beginning and then drop down to their regular value. In this way, the upper branch is excited and the pulse energy should still be modest. Because of the bistability, the "linear" and nonlinear pulses can now have the same power for the off-state as well as for the on-state. The difference between the linear and nonlinear regimes is simply due to the power peak at the start of the pulse. In this way, there is also no difference anymore between $\left|t_{\text {tot }}\right|^{2}$ and $\left|t_{\text {tot }}\right|^{2} P_{\text {in. }}$.

Until now only one period was considered. Like in Section VI-A, the contrast $C$ will raise with increasing $N$. This is also shown in Fig. 16. A contrast of one can again be obtained for $\Delta \nu_{c}=(1 / 2)\left(\Delta \nu+\Delta \nu_{s}\right)$ in the case of $\infty$ periods. However, increasing $N$ will also increase the power density required at the start of the pulse in order to switch to the upper branch, so

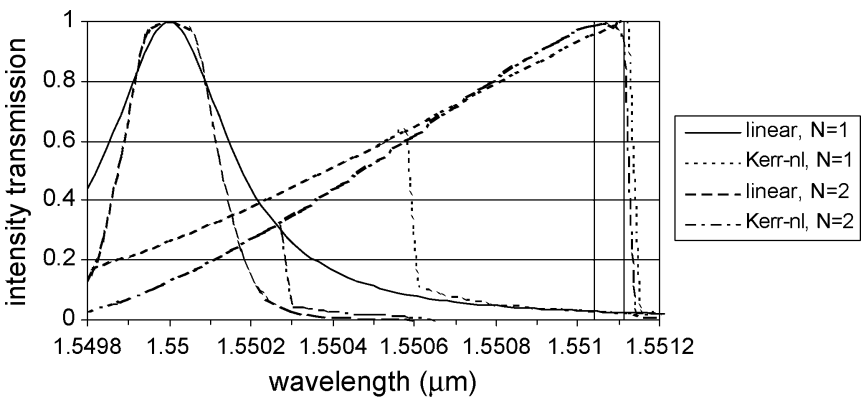

Fig. 16. Numerical calculation of the linear and nonlinear transmission $\left|t_{\text {tot }}(\lambda)\right|^{2}$ for a resonator structure consisting of one and two periods. The parameters values which are used, are given in the text. The signal bandwidth is indicated by vertical lines.

the number of periods should be kept small. Furthermore, one can see in Fig. 13 that the achievable contrast with a single period is already very high, so the relative improvement obtained by increasing the number of periods will be very small.

\section{CONCLUSION}

In this paper, the designs of the most common all-optical functionalities - all-optical phase shifting, limiting, switching, and bistability - have been investigated in case the data signal is responsible for nonlinear operation. The influence of the different parameters has been analyzed and general aspects to which one should pay attention during the design have been discussed.

In particular, it was shown that in order to make an efficient design, it is of the utmost importance to obtain a refractive index contrast in the mirrors which is as high as possible. A higher contrast does not only reduce the device length (because the mirror will be smaller for the same reflectivity), but it also substantially reduces the required input power. Furthermore, the cavity length must be kept small, although not necessarily a half-wavelength. Only in the case of all-optical limiting, the cavity length is of less importance and can be used for tuning the power output. Except for all-optical phase shifting, a small number of resonator periods is generally sufficient for a reasonable nonlinear operation.

Special attention must, however, be paid to the signal bandwidth that one would like to use. All nonlinear functionalities discussed in this paper can be achieved for reasonable input powers $(<100 \mathrm{~mW})$ and device lengths $(<250 \mu \mathrm{m})$ under the condition that the signal bandwidth is limited $(<10 \mathrm{GHz})$. Higher band rates require substantially more optical power or much longer device lengths. This means that for the moment application of nonlinear devices will typically be restricted to dense wavelength-division multiplexing systems. This restriction can only be lifted by searching for materials with higher Kerr nonlinearities.

In addition to this, it was also brought to attention that insertion of nonlinear applications into standard technologies cannot be done in a straightforward manner. In general, two different power levels will have to be taken into account (a linear and a nonlinear one), so the data processing will become more complicated. Also the used pulse shapes can be different from common practice. 
To conclude, one can say that resonating structures allow the implemention of all-optical ultrafast functionalities within reasonable power budgets and device lengths, but not without limitations. The usable bandwidth is limited and the data processing will generally be more complex.

\section{REFERENCES}

[1] G. Priem, P. Bienstman, G. Morthier, and R. Baets, "Resonator-based all-optical Kerr-nonlinear phase shifting: design and limitations," $J$. Appl. Phys., submitted for publication.

[2] A. Melloni, F. Morichetti, and M. Martinelli, "Linear and nonlinear pulse propagation in coupled resonator slow-wave optical structures," Opt. Quantum Electron., vol. 35, pp. 365-379, 2003.

[3] J. E. Heebner and R. B. Boyd, "Enhanced all-optical switching by use of a nonlinear fiber ring resonator," Opt. Lett., vol. 24, pp. 847-849, 1999.

[4] S. Blair, "Nonlinear sensitivity enhancement with 1D photonic bandgap microcavity arrays," Opt. Lett., vol. 27, pp. 613-615, 2002.

[5] M. Soljacic, S. G. Johnson, S. Fan, M. Ibanescu, E. Ippen, and J. D. Joannopoulos, "Photonic-crystal slow-light enhancement of nonlinear phase sensitivity," J. Opt. Soc. Amer. B, vol. 19, pp. 2052-2059, 2002.

[6] D. Pelinovsky, J. Sears, L. Brzozowski, and E. H. Sargent, "Stable alloptical limiting in nonlinear periodic structures. I. Analysis," J. Opt. Soc. Amer. B, vol. 19, pp. 43-53, 2002.

[7] J. He and M. Cada, "Optical bistability in semiconductor periodic structures,” IEEE J. Quantum Electron., vol. 27, pp. 1182-1188, May 1991.

[8] U. Peschel, T. Peschel, and F. Lederer, "Optimization of bistable planar resonators operated near half the band gap," IEEE J. Quantum Electron., vol. 30, pp. 1241-1249, May 1994.

[9] S. Radic, N. George, and G. Agrawal, "Theory of low-threshold optical switching in nonlinear phase-shifted periodic structures," J. Opt. Soc. Amer. B, vol. 12, pp. 671-680, 1995.

[10] J. Lee and G. P. Agrawal, "Nonlinear switching of optical pulses in fiber Bragg gratings," IEEE J. Quantum Electron., vol. 39, pp. 508-515, Mar. 2003.

[11] D. Weaire and J. P. Kermode, "Dispersive optical bistability: numerical methods and definitive results," J. Opt. Soc. Amer. B, vol. 3, pp. 1706-1711, 1986.

[12] P. W. Smith and E. H. Turner, "A bistable Fabry-Perot resonator," Appl. Phys. Lett., vol. 30, pp. 280-281, 1977.

[13] H. A. Haus and Y. Lai, "Theory of cascaded quarter wave shifted distributed feedback resonators," IEEE J. Quantum Electron., vol. 28, pp. 205-213, Jan. 1992.

[14] J. M. Bendickson and J. P. Dowling, "Analytic expressions for the electromagnetic mode density in finite, one-dimensional, photonic bandgap structures," Phys. Rev. E, vol. 53, pp. 4107-4121, 1996.

[15] H. K. Tsang, C. S. Wong, T. K. Liang, I. E. Day, S. W. Roberts, A. Harpin, J. Drake, and M. Asghari, "Optical dispersion, two-photon absorption and self-phase modulation in silicon waveguides at $1.5 \mu \mathrm{m}$ wavelength," Appl. Phys. Lett., vol. 80, pp. 416-418, 2002.

[16] B. Maes, P. Bienstman, and R. Baets, "Modeling of Kerr nonlinear photonic components with mode expansion," Opt. Quantum Electron., vol. 36, pp. 15-24, 2004.

[17] CAMFR (CAvity Modelling FRamework) home page [Online]. Available: http://camfr.sourceforge.net/

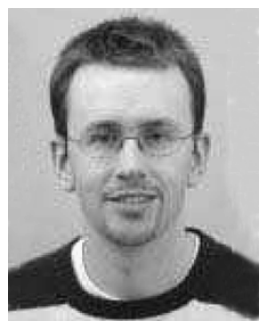

Gino Priem (S'02) was born in Veldegem, Belgium, in 1979 . He received the degree in physical engineering from Ghent University, Ghent, Belgium, in 2002. He is currently working toward the Ph.D. degree in electrical engineering at Ghent University.

His research interests include modeling, design, and fabrication of nonlinear applications by means of resonators for ultrafast all-optical signal processing.

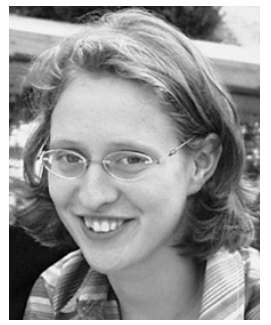

Inge Notebaert was born in Ghent, Belgium, in 1980. She received the degree in mathematics from Ghent University, Ghent, in 2002. She is currently working toward the M.Ec. degree in accounting at Ghent University.

Her research interests include mathematical modeling.

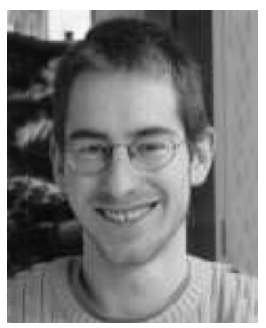

Bjorn Maes (S'04) received the degree in engineering (physics) from Ghent University, Ghent, Belgium, in 2001. He is currently working toward the Ph.D. degree in electrical engineering in the Department of Information Technology at Ghent University.

His research interests include the modeling of nonlinearities in integrated optics in general, and photonic crystals in particular.

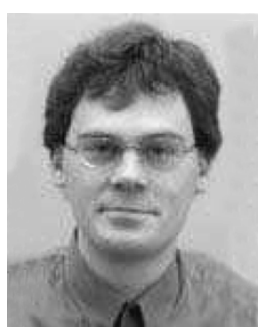

Peter Bienstman (S'97-A'01-M'03) was born in Ghent, Belgium, in 1974. He received the degree in electrical engineering and the Ph.D. degree from the Department of Information Technology (INTEC), Ghent University, Belgium, in 1997 and 2001. respectively.

During 2001-2002, he spent a year in the Joannopoulos research group at the Massachusetts Institute of Technology, Cambridge. He is currently a Postdoctoral Fellow at Ghent University. He has published several papers and has one patent. His research interests include the modeling of optical structures, notably photonic crystal structures, vertical-cavity surface-emitting lasers, and resonant-cavity light-emitting diodes.

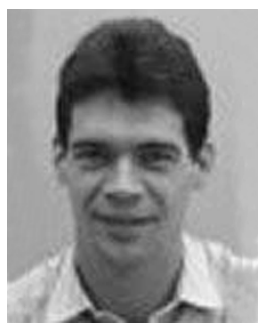

Geert Morthier (M'93-SM'01) received the degree in electrical engineering and the $\mathrm{Ph} . \mathrm{D}$. degree from Ghent University, Ghent, Belgium, in 1987 and 1991, respectively.

Since 1991, he has been a Member of the Permanent Staff of the Interuniversity MicroElectronics Center (IMEC), Ghent University. From 1998 to 1999, he was the Project Manager of the ACTS project ACTUAL, and since 2001 he has been Project Manager of the IST project NEWTON on widely tunable lasers. In 2001, he was appointed part-time Professor at Ghent University. He has authored or coauthored around 100 papers and two books in the field. His main research interests are in the modeling and characterization of optoelectronic components.

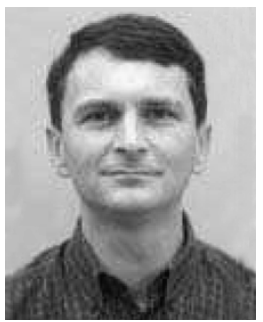

Roel Baets (M'88-SM'96) received the degree in electrical engineering and the Ph.D. degree from Ghent University, Ghent, Belgium, in 1980 and 1984, respectively, and the M.Sc. degree in electrical engineering from Stanford University, Stanford, CA, in 1981 .

Since 1981 he has been with the Department of Information Technology (INTEC), Ghent University, where he has been a Professor since 1989. He is currently the Head of the optoelectronics group. $\mathrm{He}$ has worked in the field of III-V devices for optoelectronic systems. With about 200 publications and conference papers, he has made contributions to the design and fabrication of semiconductor laser diodes, passive guided wave devices, PICs, and microoptic components.

Dr. Baets is a Member of the Optical Society of America, the IEEE Laser and Electro-Optics Society, the International Society for Optical Engineering (SPIE), and the Flemish Engineers Association. 